\title{
基于变分贝叶斯高斯混合噪声模型的机器人跨模态 生成对抗网络
}

熊鹏文 ${ }^{1,2,3^{*}}$ ，童小宝 ${ }^{1}$ ，宋爱国 ${ }^{2,3^{*}}$ ，刘小平 4

1. 南昌大学信息工程学院, 南昌 330031 , 中国

2. 东南大学仪器科学与工程学院, 南京 210096 , 中国

3. 东南大学生物电子学国家重点实验室, 南京 210096 , 中国

4. Department of Systems and Computer Engineering, Carleton University, Ottawa K1S5B6, Canada

* 通信作者. E-mail: steven.xpw@ncu.edu.cn, a.g.song@seu.edu.cn

收稿日期: 2020-05-30; 修回日期: 2020-07-19; 接受日期: 2020-08-13; 网络出版日期: 2020-12-16

国家自然科学基金 (批准号: 61903175，61663027，91648206)、国家重点研发计划“云端融合的自然交互设备和工具”(批准号： 2016 YFB1001300) 和江西省主要学科学术和技术带头人项目 (批准号: 20204BCJ23006) 资助

摘要 多模态融合对于机械手充分感知外界环境至关重要，单一模态信息会限制机械手对物体的识 别、抓取能力, 而传统的跨模态数据生成方法生成的图像效果较差, 导致多模态融合效果并不理想. 为 了解决跨模态生成的图像效果差和多模态融合的数据缺乏等问题, 本文提出变分贝叶斯高斯混合条件 生成对抗网络 (BGM-CGAN) 的跨模态多样性噪声数据生成式方法. 首先利用变分贝叶斯高斯混合算 法将均匀分布的随机噪声组生成单一的混合变量; 然后将生成的混合变量通过高斯混合模型生成一系 列高斯混合噪声组; 最后从生成的高斯混合噪声组中随机选取单一高斯噪声导入辅助模态图像中, 并 与辅助模态图像进行融合, 成功生成了高清晰度的异域模态图像, 真实还原了异构模态信息, 解决了单 一模态信息不足和生成图像质量差等问题。最后利用 Inception Score (IS)、Frechet Inception Distance (FID)、结构相似性 (SSIM)、峰值信噪比 (PSNR) 等评价体系, 将 BGM-CGAN 算法与其他算法的跨 模态生成图像能力进行对比, 验证了所提出算法的有效性和可行性. 此外, BGM-CGAN 算法还可延伸 应用于跨模态材料检索、跨模态纹理识别等领域, 具有广泛应用前景.

关键词 跨模态, 变分贝叶斯高斯混合, 条件生成对抗网络

\section{1 引言}

视觉和触觉两种信息源对于机械手感知外部环境至关重要, 其中视觉可以辨知物体的类别 $[1,2]$ 和 空间距离, 触觉可以感知物体的材料 ${ }^{[3]}$ 和硬度 ${ }^{[4]}$ 等, 所以将两种模态信息进行融合可以增强机械手

\footnotetext{
引用格式: 熊鹏文, 童小宝, 宋爱国, 等. 基于变分贝叶斯高斯混合噪声模型的机器人跨模态生成对抗网络. 中国科学: 信息科学, 2021, 51: 104-121, doi: 10.1360/SSI-2020-0153

Xiong P W, Tong X B, Song A G, et al. Robotic cross-modal generative adversarial network based on variational Bayesian Gaussian mixture noise model (in Chinese). Sci Sin Inform, 2021, 51: 104-121, doi: 10.1360/SSI-2020-0153
} 
的感知能力. 然而在现实操作环境中, 比如机械手在探索物体进行抓取操作时 [5 8] 往往会缺乏相应的 模态数据, 导致对被操作物体感知偏差, 造成操作任务失败. 因此, 基于已知模态数据生成还原度高的 未知对立模态数据至关重要. 然而截至目前, 关于跨模态数据生成式算法的研究很少, 尤其是能生成 高还原度跨模态图像的算法.

本文利用变分贝叶斯高斯混合条件生成对抗网络 (BGM-CGAN) 处理跨模态数据弥补单一模态信 息不足的缺陷. 以 UR5 机械臂 ${ }^{[9]}$ 为例, 其末端搭载有外部摄像头用于捕捉抓取物体时物体与夹持器的

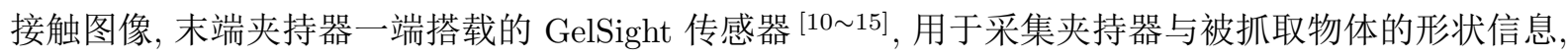
并利用图像形式表达触觉信息, 如图 1 所示. 本文正是利用文献 [9] 提供的数据集验证 BGM-CGAN 效果, 首先将收集的像素值为 $640 \times 480$ 的视觉和触觉图像进行像素预处理转换为像素值为 $256 \times 256$ 的视觉和触觉图像, 然后将预处理的视觉 - 触觉图像分别与多样贝叶斯高斯混合噪声数据进行融合, 导入到条件生成对抗网络中. 其中, 在生成视觉图像时, 将触觉图像与混合噪声结合, 利用视觉图像作 为辅助图像; 在生成触觉图像时, 将视觉图像与混合噪声结合, 利用触觉图像作为辅助图像. 具体操作 为: 首先预生成多个均匀分布的噪声组, 其次将该噪声组通过变分贝叶斯高斯混合算法生成一个高斯 混合变量, 最后将该高斯混合变量导入到高斯混合模型中, 生成多个高斯混合噪声, 并从中随机选取 一个噪声导入条件生成对抗网络, 利用重调参技巧 [16,17] 生成高质量跨模态图像. 另外, 为了直观体现 生成的跨模态图像质量, 本文还提出了单高斯条件生成对抗网络 (SGM-CGAN) 和高斯混合条件生成 对抗网络 (MOG-CGAN) 来进行跨模态数据生成对比.

\section{2 相关工作}

在生成对抗式网络 (GAN) 研究中, Erickson 等 [18] 利用 GAN 进行半监督材料识别, 并在没有标 记属性条件下取得了较好的材料识别结果. 但是材料收集过程复杂且相应的触觉传感器难以制作. 卫 星等 [19] 使用了多视角图像生成算法 (ViewGAN) 将单个视角图像生成多个视角图像, 该网络包括多 个生成器和一个多类别判别器, 可灵活迁移至多视角生成的多个场景, 但是该算法训练过程复杂, 且 生成的图像容易出现失真等情况. 栗梦媛等 ${ }^{[20]}$ 提出基于 GAN 的回归无线定位方法, 从海量数据中 提取定位特征, 避免了人为指定特征而导致的低精度问题, 但是采用预训练的 GAN, 实验稳定性不足.

在跨模态生成数据和识别物体研究中, Lee 等 [21] 利用随机均匀噪声结合条件生成对抗网络 (CGAN) 跨模态感知材料特性, 通过该方法生成了对立模态数据, 提高了视觉 - 触觉融合材料识别 结果. 但是由于对立模态数据与原本模态数据差异性极大, 随机均匀噪声难以刻画对立模态图像像素 值, 所以导致生成的跨模态图像质量较低. Falco 等 ${ }^{[22]}$ 采用潜在空间联合表征方式进行视觉 - 触觉 相关性探索, 利用视觉模态建立训练模型, 然后通过同一类别视觉 - 触觉潜在空间变量具有部分相似 性这一条件将触觉模态导入到由视觉模态训练的模型中进行跨模态物体识别. 但是由于视觉 - 触觉 差异性极大, 导致潜在表征相似度很小, 所以这种跨模态物体识别效果较差. Liu 等 ${ }^{[23]}$ 利用判别对 抗网络进行跨模态材料检索研究, 通过引入相同模态间判别损失、相同模态间对抗损失和不同模态间 变量损失探索视觉 - 触觉间相同表征特性, 并取得了较好的检索结果, 但是这种方法计算量较大且耗 时. $\mathrm{Li}$ 等 ${ }^{[24]}$ 通过残差网络提取视觉 - 触觉信息, 引入了视觉序列导入到输入空间和对抗空间, 克 服了视觉模态虽然可以感知整个物体, 但存在时间上的缺陷而无法直接应用于滑动检测等研究场景, 极大提高了跨模态图像生成效果. 但是此实验依赖于大规模数据集, 并需要视觉序列等繁杂条件. $\mathrm{Li}$ 等 ${ }^{[25]}$ 使用集成生成对抗网络学习视觉 - 触觉表征, 并提取原视觉图像特征生成潜在矢量, 与噪声生 成的频谱图融合生成触觉时间序列信号, 实现了从视觉图像到触觉序列的新颖生成方式, 为跨模态材 


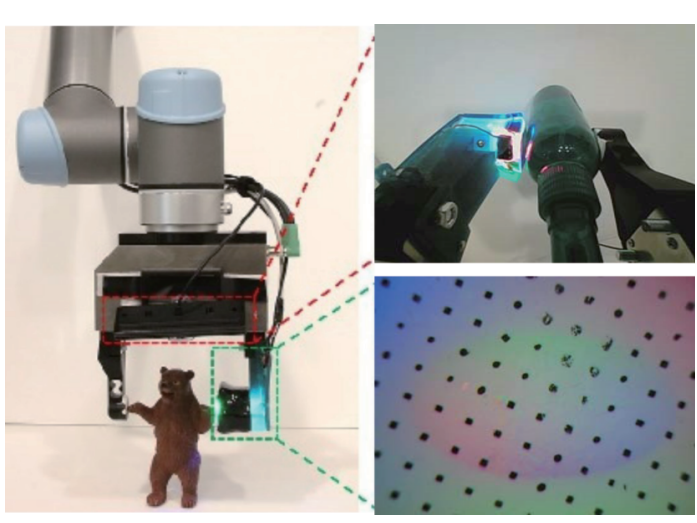

图 1 视觉 - 触觉模态数据收集过程

Figure 1 Visual-tactile modal data collection process

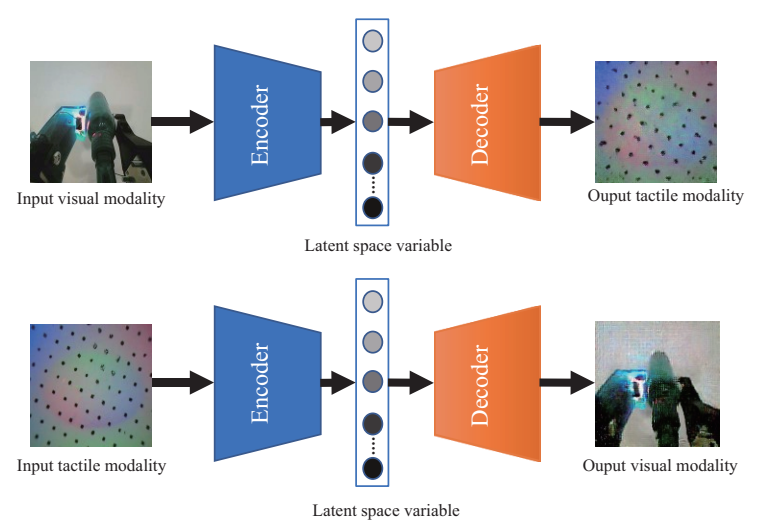

图 2 生成器编码解码过程

Figure 2 Generator encoder and decoder process

料检索研究提供了新方法. 但是此方法在生成频谱图的算法中, 采用的是 CGAN 模型, 即输入的噪声 信号为单一的满足随机均匀分布的噪声, 很难完全刻画频谱图这样具有像素多样性特征的图像, 从该 文中可以看出原始频谱图像和生成的频谱图像具有一定差异性. Luo 等 ${ }^{[26]}$ 利用卷积网络分别对触觉 模态和视觉模态进行隐藏层特征提取, 然后引入了最大协方差分析 MCA 来探索隐藏层视觉 - 触觉共 同特征, 从而利用触觉 - 视觉间隐藏层特征相似性进行跨模态衣物纹理识别, 所以此方法主要是利用 对立模态潜在空间特征相似性来进行跨模态识别研究. Sun 等 ${ }^{[27]}$ 使用跨模态零样本算法通过稀疏编 码将已知的视觉 - 触觉物体类别数据进行一一对应匹配训练, 并且利用同种材料类别触觉序列间数据 相似性, 从而探索到未标记的触觉数据的具体视觉物体类别, 属于一种跨模态物体识别研究. 为了解决 在不同模态条件下生成相应的跨模态辅助图像模糊、塌陷等问题, 本文提出 BGM-CGAN, 利用输入噪 声数据的多样性和约束性特征刻画跨模态图像的像素复杂性特征 (因为多个满足正态分布的高斯噪声 组件可以生成任意跨模态图像像素多样性特征, 并且在迭代过程中, 本文使用 RMSPropOptimizer 优 化器更新了噪声组件的均值和方差参数, 使生成的图像同时向原始图像收玫), 能够生成清晰且还原度 高的跨模态数据. 如图 2 为 BGM-CGAN 生成器视觉 - 触觉和触觉 - 视觉两种不同形式的跨模态编 码解码过程. 本文贡献体现在下述 3 方面.

(1) 现有的跨模态生成式算法将随机均匀噪声输入到 CGAN, 生成的跨模态图像失真度高. 针对 这一问题, 本文将变分贝叶斯高斯混合噪声模型获取的随机混合噪声输入到 CGAN, 通过混合噪声输 入方式, 可以刻画对立模态生成图像的多样性特征, 获取还原度高的跨模态图像.

(2) 为了体现 BGM-CGAN 算法优越性, 本文还提出了 SGM-CGAN 和 MOG-CGAN, 其中 SGMCGAN 将输入到 CGAN 的随机均匀噪声替换为符合高斯分布的单高斯噪声; MOG-CGAN 在 SGMCGAN 基础上改进了噪声输入模式, 将单高斯噪声替换为具有多个高斯组件的混合噪声模式, 因为理 论上, 多个高斯混合模式是可以刻画任意图像像素特征的. 同时, 本文对所提出的这两个网络采用重 调参技巧 $[16,17]$ 更新高斯组件参数, 提高生成图像清晰度.

(3) 采用跨模态生成方式将单一模态感知方式转换为多模态感知, 为视觉 - 触觉多模态融合研究提 供了新的方案支持; 采用 Inception Score (IS)、Frechet Inception Distance (FID)、结构相似性 (SSIM)、 峰值信噪比 (PSNR) 等多种评价体系, 对 BGM-CGAN 等算法性能进行了定量、定性评估. 


\section{GAN 的相关背景}

随着深度学习技术的发展, 图像生成研究已经成为一个热门方向, 尤其在生成高质量图像方面. 变 分自编码器 (VAE) ${ }^{[17]}$ 是一种基于概率图的生成算法, 引入重调参技巧提高了模型生成图像能力. 之 后研究人员又将 VAE 模型改进为基于注意力机制的 DRAW 模型, 进一步提高了生成图像的质量. 而 最近研究中, 人们越来越关注 GAN 的图像生成能力, 并在此基础上提出了很多改进的新型 GAN, 如 $\mathrm{CGAN}^{[28]}$, BiGANs ${ }^{[29]}$, InfoGANs ${ }^{[30]}$ 等.

GAN 主要包括两个学习网络: 生成器和判别器. 其中生成器由编码器和解码器组成, 编码器将原 始图像解码到潜在空间, 生成潜在矢量, 而解码器将潜在矢量重组为生成图像. 判别器用来判别生成 图像是真实样本还是虚假样本, 主要基于最大最小博亦理论同时进行网络训练. 若输入到判别器的样 本为真实样本, 则输出为 1 ; 若输入为虚假样本, 则输出为 0 . 而生成器的目标就是达到以假乱真的目 的, 使判别器对生成器生成的虚假图像难以区分. 通用的 GAN 的生成器目标损失函数表示为

$$
\min V_{G}(D, G)=\min \left(E_{z \sim p_{z}}(\log (1-D(G(z))))\right) .
$$

判别器目标损失函数表示为

$$
\min V_{D}(D, G)=\min \left\{E_{x \sim p_{\text {data }}}(\log D(x))+E_{z \sim p_{z}}(\log (1-D(G(z))))\right\}
$$

最终整个生成对抗网络 GAN 的目标损失函数为

$$
\min \max V(D, G)=\min \max \left\{E_{x \sim p_{\text {data }}}(\log D(x))+E_{z \sim p_{z}}(\log (1-D(G(z))))\right\} .
$$

尽管 GAN 在图像生成能力方面具有很高的效率, 但是本文旨在讨论跨领域图像生成能力, 显然 GAN 难以实现跨领域生成图像, 故进一步引进 CGAN 进行跨领域图像生成研究. 事实上, CGAN 只在 GAN 基础上增加了条件项 $y$, 这个条件可以为任意形式, 本文将条件项 $y$ 特定为辅助模态. 与 GAN 相比, 通用的 CGAN 模型的总体损失函数为

$$
\min \max V(D, G)=\min \max \left\{E_{x \sim p_{\text {data }}}(\log D(x \mid y))+E_{z \sim p_{z}}(\log (1-D(G(z \mid y))))\right\}
$$

该基础模型输入的噪声为随机均匀噪声, 其满足的概率密度函数为

$$
f(x)=\left\{\begin{array}{l}
\frac{1}{b-a}, a \leqslant x<b, \\
0, \text { else }
\end{array}\right.
$$

其中 $b$ 为均匀分布的下界, $a$ 为均匀分布的上界.

\section{BGM-CGAN 模型}

本文在提出 BGM-CGAN 算法之前, 先介绍基于高斯噪声的跨模态条件生成对抗网络 SGM-CGAN 算法和 MOG-CGAN 算法. 


\subsection{SGM-CGAN}

本文引进的高斯模型分为单高斯噪声模型和高斯混合噪声模型, 其中高斯混合噪声模型是由多个 单高斯模型组合而成的. 而单高斯噪声模型对比上述提到的随机均匀噪声模型, 其满足的噪声概率密 度函数如下式:

$$
f(x)=\frac{1}{\sqrt{2 \pi} \sigma} \exp \left(\frac{(x-\mu)^{2}}{2 \sigma^{2}}\right) .
$$

使 $f(x)=N(\mu, \sigma)$, 其中 $\mu$ 为单高斯噪声的样本均值, $\sigma$ 为样本方差. 为提升跨模态生成图像品质, 同时使 CGAN 便于计算高斯参数, 本文引进了重调参技术, 使单高斯噪声 $z=\mu+\sigma \varepsilon$, 其中高斯参数 $\varepsilon \sim N(0,1)$. 这里将单高斯噪声融合到 CGAN 网络的生成器结构, 因此接下来讨论 CGAN 的生成 器 $G$ 生成的样本分布 $p_{\text {data }}(G(z))$, 其目标是尽可能地描述真实样本分布, 为了简化, 使 $z=z \mid y$, 则 $p_{\text {data }}(G(z))$ 可表达为

$$
p_{\text {data }}(G(z))=\int_{z} p(G(z), z) \mathrm{d} z=\int_{z} p_{\text {data }}(G(z) \mid z) p_{z}(z) \mathrm{d} z,
$$

其中 $p_{z}(z)$ 为先验分布密度函数, 使 $z=\mu+\sigma \varepsilon$, 生成器生成的样本分布 $p_{\text {data }}(G(z))$ 可由下式计算:

$$
p_{\text {data }}(G(z))=\int_{z} p_{\text {data }}(G(\mu+\sigma \varepsilon) \mid z) p_{z}(z) \mathrm{d} z .
$$

这里, 为了防止单高斯噪声的样本方差参数 $\sigma$ 在参数更新过程中变为 0 , 故在生成器损失函数中添加 $l_{2}$ 正则化项, 则最终基于单高斯噪声的跨模态生成对抗网络 SGM-CGAN 的生成器 $G$ 损失函数为

$$
\min V_{G}(D, G)=E_{z \sim p_{z}}(\log (1-D(G(z))))+\lambda(1-\sigma)^{2} .
$$

\subsection{MOG-CGAN}

高斯混合噪声模型是由多个单高斯噪声模型组合而成的, 生成的图像受到多个高斯组件的共同约 束, 具备多样性特征, 更容易刻画跨领域图像这种异质图像特征对, 生成的图像效果不易出现崩塌、失 真等问题. 设有 $N$ 个单高斯噪声组件来生成跨模态图像, 其混合样本均值组 $\mu=\left[\mu_{1}, \mu_{2}, \ldots, \mu_{N}\right]$, 混 合样本方差组为 $\Sigma=\left[\Sigma_{1}, \Sigma_{2}, \ldots, \Sigma_{N}\right]$, 则其满足的先验分布的密度函数为

$$
p_{z}(z)=\sum_{i=1}^{N} \phi_{i} g\left(z \mid \mu_{i}, \Sigma_{i}\right),
$$

其中 $\phi_{i}$ 代表每个单高斯组件在相应高斯混合模型的权重, $g\left(z \mid \mu_{i}, \Sigma_{i}\right)$ 代表单个高斯噪声组件的概率密 度函数. 理论上, $\phi_{i}, \mu_{i}, \Sigma_{i}$ 可以通过 $\mathrm{EM}$ 算法 ${ }^{[31]}$ 迭代算出相应各个高斯分量参数, 但由于本文是将 高斯混合噪声导入到 CGAN 网络中, 无法通过计算更新各个高斯分量的权重 $\phi_{i}$, 所以使每个高斯噪 声分量的权重相等, 采用随机选择的方式选择任意高斯噪声分量导入 CGAN 的生成器中, 与辅助模态 进行融合. 设 $\phi_{i}=1 / N$ 则满足的先验分布的密度函数可以写为

$$
p_{z}(z)=\sum_{i=1}^{N} \frac{g\left(z \mid \mu_{i}, \Sigma_{i}\right)}{N} .
$$

利用重调参技巧, $\Sigma_{i}=\sigma_{i}$, 使 $z=\mu_{i}+\sigma_{i} \varepsilon$, 则 MOG-CGAN 生成器 $G$ 生成的样本概率分布函数可以 写为

$$
p_{\text {data }}(G(z))=\sum_{i=1}^{N} \int \frac{p_{\text {data }}\left(G\left(\mu_{i}+\sigma_{i} \varepsilon\right) \mid \varepsilon\right) p(\varepsilon) \mathrm{d} \varepsilon}{N},
$$

108 
并且再次引入了 $l_{2}$ 正则化项, 防止 $\sigma_{i}$ 更新过程中衰减至 0 . 通过以上分析, 基于 MOG-CGAN 的生成 器 $G$ 的损失函数可以写为

$$
\min V_{G}(D, G)=\min \left(E_{z \sim p_{z}}(\log (1-D(G(z))))\right)+\lambda \sum_{i=1}^{N} \frac{\left(1-\sigma_{i}\right)^{2}}{N} .
$$

初始化 $\mu_{i}$ 满足随机均匀分布 $[-1,1], \sigma_{i}=0.2, \varepsilon$ 满足高斯分布 $N(0,1)$, 并且参数 $\mu_{i}$ 和 $\sigma_{i}$ 会随着迭 代在 RMSPropOptimizer 优化器中进行参数更新, 则 MOG-CGAN 网络总体损失函数为

$$
\min \max V(D, G)=\min \max \left\{E_{x \sim p_{\text {data }}}(\log D(x))+\min V_{G}(D, G)\right\},
$$

其中高斯混合噪声包括了 $N$ 个单高斯噪声组件, 每个单高斯噪声组件满足不同的样本均值和方差, 但 满足相同的高斯参数 $\varepsilon$. 训练过程中, 每次随机选取一个单高斯噪声组件融合到辅助模态中, 通过 $N$ 个高斯噪声源可以刻画跨域生成图像的多样性特征.

\subsection{BGM-CGAN}

本文提出的 BGM-CGAN 是在 MOG-CGAN 基础上进行的参数优化, 使原来 MOG-CGAN 中满 足单高斯分布的参数 $\varepsilon$ 改变为由多个均匀随机噪声经过变分贝叶斯高斯混合分布得来的混合参数 $\varepsilon$, 使得最终输入到 CGAN 生成器的高斯混合噪声组满足双重约束条件: 第一, 高斯混合噪声组的样本均 值参数 $\mu_{i}$ 是由随机均匀噪声组件随机选取的; 第二, 高斯混合噪声组的高斯参数 $\varepsilon$ 是由随机均匀噪 声组件经过变分贝叶斯高斯混合模型计算得出的, 使得 BGM-CGAN 在判别器 $D$ 优化器中优化参数 $\mu_{i}$ 时, 也同时优化了混合高斯参数 $\varepsilon$, 相比 MOG-CGAN 网络的随机高斯参数 $\varepsilon$ 更具有约束性和回归 性. 首先随机均匀噪声组件的混合高斯概率密度为

$$
p(x, z, \pi, \mu, \sigma)=\int_{z} p(x \mid z, \mu, \sigma) p(z \mid \pi) p(\pi) p(\mu \mid \sigma) p(\sigma) \mathrm{d} z .
$$

由于隐变量 $z$ 的概率分布比较难求, 故采用变分法来近似求解复杂的概率分布 $p$, 在变分法中, 采用简 单的分布 $q$ 去近似复杂分布 $p, q$ 的分解特性使其能在变分 EM 算法中更新相应混合参数, 如下式:

$$
q(z, \pi, \mu, \sigma)=q(z) q(\pi, \mu, \sigma),
$$

其中 $q(z)$ 和 $q(\pi, \mu, \sigma)$ 可以用变分 $\mathrm{EM}$ 推断求解, 其中使

$$
\begin{aligned}
& \ln q(z)=E_{\pi, \mu, \sigma}[\ln p(x, z, \pi, \mu, \sigma)]+\mathrm{C} 1, \\
& \ln q(\pi, \mu, \sigma)=E_{z}[\ln p(x, z, \pi, \mu, \sigma)]+\mathrm{C} 2,
\end{aligned}
$$

将上式转化为

$$
\ln q(\pi, \mu, \sigma)=\ln q(\pi) \ln q(\mu, \sigma),
$$

而 $\ln q(\pi)$ 可以使狄里克利分布自适应其相应随机均匀噪声权重, $\alpha$ 为混合参数 $\pi$ 的先验分布矢量, 如 下式:

$$
q\left(\pi_{k}\right)=\operatorname{Dir}\left(\pi_{k} \mid \alpha_{k}\right) .
$$

而相应混合分布的参数 $\mu$ 更新可通过下式:

$$
q\left(\mu_{k} \mid \sigma_{k}\right)=N\left(\mu_{k} \mid m_{k},\left(\beta_{k} \sigma_{k}\right)^{-1}\right),
$$


其中参数 $\beta$ 和 $m$ 更新如下:

$$
\begin{gathered}
\beta_{k}=\beta_{0}+N_{k}, \\
m_{k}=\frac{\beta_{0} m_{0}+N_{k} \hat{y_{k}}}{\beta_{0}+N_{k}} .
\end{gathered}
$$

参数 $\sigma$ 更新如下:

$$
\begin{gathered}
q\left(\sigma_{k}\right)=W\left(\sigma_{k} \mid \nu_{k}, W_{k}\right), \\
\nu_{k}=\nu_{0}+N_{k}, \\
W_{k}^{-1}=W_{0}^{-1}+N_{k} S_{k}+\frac{\beta_{0} N_{k}}{\beta_{0}+N_{k}}\left(\hat{y_{k}}-m_{0}\right)\left(\hat{y_{k}}-m_{0}\right)^{\mathrm{T}} .
\end{gathered}
$$

以上参数更新通过变分 $\mathrm{EM}$ 推导. 经过变分贝叶斯高斯混合估计获取可得到的随机混合参数 $\varepsilon$, 然后将参数 $\varepsilon$ 导入到 MOG-CGAN 网络中. 图 3 所示为 BGM-CGAN 跨模态生成图像训练过程. 由于 BGM-CGAN 模型中输入噪声组件的多样混合性特征和回归约束性特征, 故从图 3 的 BGM-CGAN 触 觉跨模态生成视觉图像结果看, 最主要的机械手抓取物体判断是否滑落的部分图像信息被还原出来, 从还原的跨模态视觉图像中可判断该图像表达的是稳定抓取, 且从放大的视觉图像上看, 其他抓取背 景信息基本也被真实刻画，比如机械手灯光和被抓取的黑色玻璃瓶形状等图像信息; 从 BGM-CGAN 视觉跨模态生成触觉结果看, 基本将触觉图像的形状凸显部分还原出来, 并且也真实刻画了触觉图像 各个拐角处的颜色像素值, 且还原出的像素值不像上述其他算法那样出现像素塌陷、紊乱、重度还原 等现象. 可见, 提出的 BGM-CGAN 算法一定程度上改善了跨模态生成图像的质量, 且生成的图像很 少出现崩塌、失真等现象.

\section{5 实验与分析}

\section{1 抓取数据集}

本文使用的数据集为滑动检测数据集 Gelsight-external grasp datasets ${ }^{[5]}$, 该数据集的构建方式 为: 一共抓取 100 个物体, 每个物体包括两种模态图像 (视觉模态和触觉模态), 每张图像像素值为 $640 \times 480$, 平均每个物体记录抓取状态 (抓取滑落和抓取稳定) 不少于 15 次, 每次抓取状态不少于 20 帧时序图像. 由于本文提出的模型暂未对具有变化的时帧图像进行生成处理, 所以实验只对抓取稳 定状态时帧图像进行生成 (因为大部分物体抓取稳定状态图像没有发生相对位移变化), 并且只对抓 取稳定最后 5 个时步状态进行跨模态图像生成. 最终实验结果在设备 LAPTOP-0VKE2AD9 上进行, 此电脑设备硬件配置处理器为 Intel(R) Core(TM) i7-8750 CPU @2.20 GHz, 并带有 16.0 GB 的运行 RAM, 代码主要在 Pycharm2019.2.3 上运行完成.

\section{2 算法模型}

为了验证本文提出的 BGM-CGAN 算法相对其他算法 ${ }^{[16,21]}$ 跨模态生成能力更加优越, 将与 CGAN, SGM-CGAN 和 MOG-CGAN 等算法进行跨模态生成能力对比. 这几种算法的具体实现过 程可总结如下. CGAN: 基本的跨模态生成网络, 输入噪声满足随机均匀分布, 通过更改条件项 $y$, 可以 得到相应的对立模态图像. SGM-CGAN: 通过将输入到 CGAN 生成器 $G$ 的随机均匀噪声模式改成满 足单高斯函数概率分布模式, 使单高斯噪声 $z=\mu+\sigma \varepsilon$, 并引入重调参技巧, 对相应的高斯参数进行优 化. MOG-CGAN: 在 SGM-CGAN 网络基础上将单高斯噪声改进为高斯混合噪声组, 并通过随机选择 


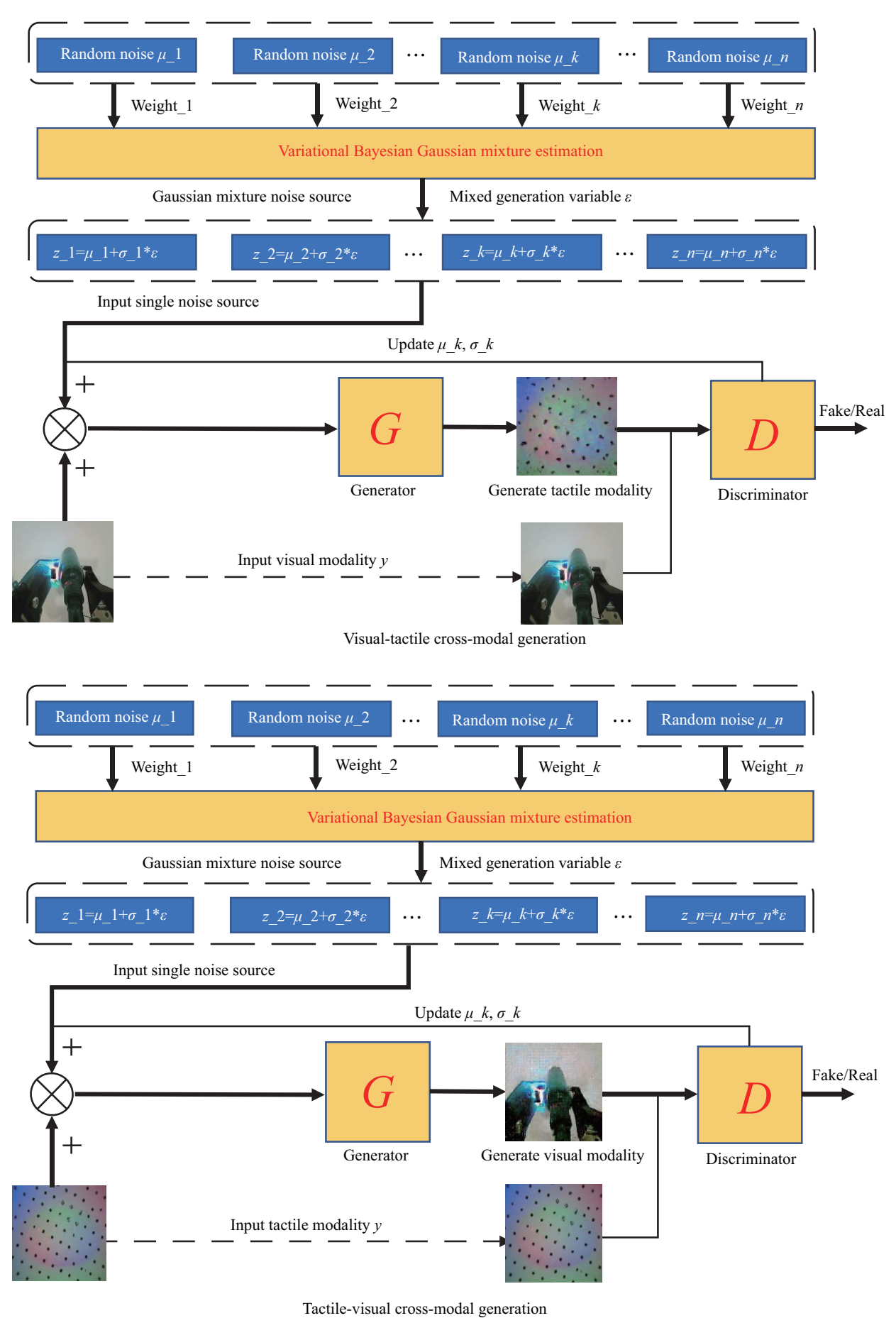

图 3 贝叶斯高斯混合网络 BGM-CGAN 跨模态生成图像过程

Figure 3 BGM-CGAN cross-modal image generation process with Bayesian Gaussian mixture noise

方式从高斯混合噪声组中选择一个高斯噪声组件与辅助模态图像融合输入到 CGAN 的生成器 $G$ 中, 通过混合噪声的多样性刻画跨域生成模型的异构性, 同时保证了生成新样本的多样性和与原样本的相 似性. BGM-CGAN: 基于 MOG-CGAN 再次丰富输入噪声的性质, 在重调参技巧基础上将相应的高斯 
混合参数中满足单高斯分布的 $\varepsilon$ 参数改进为满足变分贝叶斯高斯混合分布的 $\varepsilon$, 使 $\varepsilon$ 参数不但可由生 成高斯混合噪声组的随机均匀噪声组混合而成, 而且也能在判别器 $D$ 中进行回归优化.

\section{3 定量评估}

为了评估 BGM-CGAN 相对于其他算法模型的生成效果和算法执行复杂度, 本文采用定量评估方 法: IS, FID, SSIM 和 PSNR 评估生成图像, 并且测试各个生成式算法每迭代 100 次所需要的时间, 以 评估算法的复杂度. 其中 IS 评估方法利用 Inception V3 网络, 在 ImageNet 数据集基础上, 通过预测生 成图像中具体的物体类别, 评估生成的跨模态图像的质量和多样性, 其中 $p(y \mid x), p(y)$ 项分别体现其质 量和多样性特征. 但是此评价方法是利用 ImageNet 数据集对生成图像分类, 如果生成的跨模态图像 包含的物体类别与该数据集里包含的类别不相符, 则评估方法不能较好地评估生成图像的特征. FID 评估方法采用输出特征评估生成图像的质量和多样性, 首先通过 Inception V3 网络提取真实图像和生 成图像的特征, 再去求解两个特征之间的距离, $\mu$ 项代表该特征的均值, $\Sigma$ 项代表该特征的协方差, $r$ 下 标代表真实模态, $g$ 下标代表生成模态. 上述两种评价方法主要针对跨模态生成图像的质量和多样性 进行评估. 而 SSIM 评估方法是评估跨模态生成图像与真实图像的对比度、亮度和结构相似性, 其中 $l(x, y)$ 项是生成图像与真实图像的亮度比较, $c(x, y)$ 项是生成图像与真实图像的对比度比较, $s(x, y)$ 项是生成图像和真实图像的结构相似性比较, 故 $\operatorname{SSIM}(x, y)$ 可以反映两幅对比图像的亮度、对比度和 结构相似性的总体特征. PSNR 方法是针对不同噪声模式对生成的跨模态图像的影响进行评估的, 首 先求解真实图像与噪声生成的图像像素之间的差值, 然后将图像最大像素值平方与这种生成图像与真 实图像像素间的差异性进行对比, 从而利用计算到的到达噪声比率的顶点信号评估合成图像相对于真 实图像的质量. 最后本文为了检验算法的执行复杂度情况, 测试了各个算法迭代 100 次所需要执行的 时间.

\subsubsection{IS 评价}

为了评估生成图像的质量和多样性, 采用 inception network 对生成的图像进行分类:

$$
\mathrm{IS}=\exp \left(E_{x \sim p_{g}} D_{\mathrm{KL}}(p(y \mid x) \| p(y))\right)
$$

其中 $p(y \mid x)$ 代表生成图像的质量, $p(y \mid x)$ 值越大, 其生成的图像质量越高. $p(y)$ 反映生成图像的多样 性, $p(y)$ 值越小, 代表生成的图像多样性越高. $D_{\mathrm{KL}}$ 代表 $\mathrm{KL}$ 散度, 用来衡量两个分布之间的距离, $\mathrm{KL}$ 值越大, 代表生成图像的质量和多样性也就越高. 采用 IS 分数计算各个跨模态生成式算法模型生成 图像的质量和多样性分数, 结果如表 1 所示.

从表 1 的结果得出, 对于视觉 - 触觉和触觉 - 视觉两种跨模态生成图像模式, 提出的 BGM-CGAN 算法生成的图像效果略优于其他跨模态生成式算法, 直接证明了变分贝叶斯高斯混合噪声组件的多样 性和约束性特征能更有效地刻画生成模态图像, 而由于随机均匀噪声的随机性和其分布特征比较单一, 故以其作为输入图像生成的相应的跨模态图像效果略低于 BGM-CGAN 算法. 但是从总体的 IS 值表 现来看, 各算法 IS 值普遍较低, 说明了直接在 ImageNet 数据集基础上评估生成的跨模态图像没有考 虑到图像内容类别不完备的影响, 从而局限了对生成的图像多样性和质量的评估; 并且从表中的结果 发现 CGAN 视觉 - 触觉 IS 分数比 SGM-CGAN 算法高一点, 这可能的原因是 IS 分数并不能完全表 达生成图像的特征. 
表 1 各算法 IS 对比

Table 1 IS comparison of various algorithms

\begin{tabular}{ccc}
\hline Algorithm & Visual-tactile & Tactile-visual \\
\hline CGAN & 1.02 & 1.12 \\
SGM-CGAN & 1.01 & 1.19 \\
MOG-CGAN & 1.02 & 1.36 \\
BGM-CGAN & 1.05 & 1.60 \\
\hline
\end{tabular}

表 2 各算法 FID 对比

Table 2 FID comparison of various algorithms

\begin{tabular}{ccc}
\hline Algorithm & Visual-tactile & Tactile-visual \\
\hline CGAN & 1.99 & 4.25 \\
SGM-CGAN & 2.57 & 4.12 \\
MOG-CGAN & 2.30 & 4.43 \\
BGM-CGAN & 2.32 & 3.69 \\
\hline
\end{tabular}

\subsubsection{FID 评价}

IS 评估生成图像的质量和多样性具有一定局限性, 因为只在生成图像基础上进行图像评估, 并未 跟真实图像进行对比, 所以无法反映生成图像与真实图像的差别. FID 定量评估准则用于评估生成图 像与真实图像的在特征空间的距离特征, 故 FID 值越小, 说明其跨模态生成的样本图像真实性越高:

$$
\mathrm{FID}=\left\|\mu_{r}-\mu_{g}\right\|^{2}+\operatorname{tr}\left(\Sigma_{r}+\Sigma_{g}-2\left(\Sigma_{r} \Sigma_{g}\right)^{\frac{1}{2}}\right)
$$

其中 $\mu_{r}$ 代表真实模态的均值, $\mu_{g}$ 代表生成模态的均值, $\Sigma_{r}$ 代表真实模态的协方差, $\Sigma_{g}$ 代表生成模态 的协方差, $\operatorname{tr}$ 代表迹运算. 采用 FID 分数计算各个跨模态生成式算法模型生成图像的质量和多样性分 数, 结果如表 2 所示.

从表 2 的 FID 数据得知, 本文提出的 BGM-CGAN 算法对于触觉 - 视觉跨模态生成效果明显优 于其他算法, 说明将生成的跨模态图像与真实图像进行对比后, 更能反映 BGM-CGAN 算法生成跨模 态异构图像的能力, 并且从总体上看, 各算法的 FID 值也比较自然, 直接说明了采用输出特征进行评估 生成图像的多样性和质量相对于采用输出类别进行评估更能体现图像特征; 但是 BGM-CGAN 算法的 视觉 - 触觉跨模态生成实验效果相比 CGAN 算法生成效果略差, 这可能的原因有: 第一, BGM-CGAN 算法是一种基于双重高斯混合噪声算法, 在相同的训练迭代次数下, 噪声收玫效果没有单一均匀随机 噪声收玫效果好, 若加大 BGM-CGAN 算法迭代次数, 视觉 - 触觉跨模态生成效果将优于 CGAN; 第 二, 由于 BGM-CGAN 算法生成数据的多样性特征, 虽然可以刻画异构图像的像素特点, 但对于触觉 图像而言, 由于在抓取物体时发生形变, 部分形变信息会在刻画多样性像素特征时被忽略.

\subsubsection{SSIM 评价}

上述 IS 和 FID 评估方法是针对图像的质量和多样性进行评估的, 并不能完全体现生成图像的综 合特征. 所以采用 SSIM 方法进一步评估亮度、对比度和结构相似性, 从而评价生成图像和真实图像 之间的相似度, 其评估值范围为 $[0,1]$, SSIM 值越大代表跨模态生成图像与真实图像相比失真度越小, 
表 3 各算法 SSIM 对比

Table 3 SSIM comparison of various algorithms

\begin{tabular}{ccc}
\hline Algorithm & Visual-tactile & Tactile-visual \\
\hline CGAN & 0.94 & 0.76 \\
SGM-CGAN & 0.94 & 0.74 \\
MOG-CGAN & 0.93 & 0.75 \\
BGM-CGAN & 0.93 & 0.78 \\
\hline
\end{tabular}

说明该算法生成的跨模态生成图像质量越高:

$$
\begin{gathered}
l(x, y)=\frac{2 \mu_{x} \mu_{y}+c 1}{\left(\mu_{x}\right)^{2}+\left(\mu_{y}\right)^{2}+c 1}, \\
c(x, y)=\frac{2 \sigma_{x} \sigma_{y}+c 2}{\left(\sigma_{x}\right)^{2}+\left(\sigma_{y}\right)^{2}+c 2}, \\
s(x, y)=\frac{\sigma_{x y}+c 3}{\sigma_{x} \sigma_{y}+c 3}, \\
\operatorname{SSIM}(x, y)=\frac{\left(2 \mu_{x} \mu_{y}+c 1\right)\left(2 \sigma_{x y}+c 2\right)}{\left(\left(\mu_{x}\right)^{2}+\left(\mu_{y}\right)^{2}+c 1\right)\left(\left(\sigma_{x}\right)^{2}+\left(\sigma_{y}\right)^{2}+c 2\right)},
\end{gathered}
$$

其中 $l(x, y)$ 是生成图像与真实图像的亮度比较, $c(x, y)$ 是生成图像与真实图像的对比度比较, $s(x, y)$ 是生成图像与真实图像的结构相似度比较, $\mu_{x}$ 和 $\mu_{y}$ 分布是生成图像与真实图像的平均值, $\sigma_{x}$ 和 $\sigma_{y}$ 是其方差, $c 1$ 和 $c 2$ 的作用是防止分母为 0 . 采用 SSIM 分数计算各个跨模态生成式算法模型生成图像 的亮度、对比度和结构相似度综合分数 (SSIM 为 3 种评价指标的乘积), 结果如表 3 所示.

从表 3 的结果得知, 在触觉 - 视觉跨模态生成图像与真实图像亮度、对比度和结构比较中, BGMCGAN 算法相比其他算法得分略高, 说明在还原跨模态图像过程中, 由于 BGM-CGAN 算法中变分贝 叶斯高斯混合噪声的多样性和约束性特征, 其生成的图像失真度最小, 并且其生成的图像接近于真实 图像的亮度、对比度和结构等特点, 说明该算法在跨模态生成视觉图像任务中能取得较好的效果. 并 且从表中可以发现, 由于触觉图像的像素复杂性较低, 而视觉图像的像素复杂性较高, 所以混合噪声 模式更能刻画视觉 - 触觉跨模态这种较大差异性像素变换过程, SSIM 得分平均为 0.94 ; 而触觉 - 视 觉的跨模态生成效果较差, 可能是由于视觉图像相对于触觉图像更难还原其真实结构, 所以 SSIM 平 均值为 0.76 .

\subsubsection{PSNR 评价}

上述定量评估方法已经评估了生成图像的质量、多样性、亮度和对比度等综合因素, 接下来利用 PSNR 评估生成图像最大可接受的可能噪声功率和影响它生成质量的破坏性噪声功率的比值. 其具体 计算公式如下:

$$
\begin{gathered}
\operatorname{mse}\left(I_{g}, I_{v}\right)=\frac{1}{n} \sum_{i=1}^{n}\left(I_{v}[i]-I_{g}[i]\right)^{2}, \\
\mathrm{PSNR}=10 \log _{10}\left(\frac{\max _{I_{g}}{ }^{2}}{\mathrm{mse}}\right),
\end{gathered}
$$

其中 $\max _{I_{g}}$ 代表图像颜色的最大像素值, 这里为 RGB 图片, 故 $\max _{I_{g}}=255$, 其中 mse 代表生成图 像与真实图像间的均方误差, mse 值越小, 则 PSNR 值越大, 表示生成图像的质量越高. 为了探讨各 


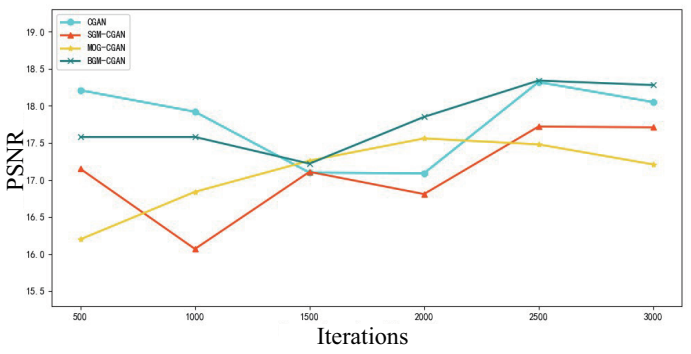

图 4 视觉 - 触觉跨模态 PSNR 值随迭代次数改变 结果

Figure 4 The visual-tactile cross-modal PSNR value changes with the number of iterations

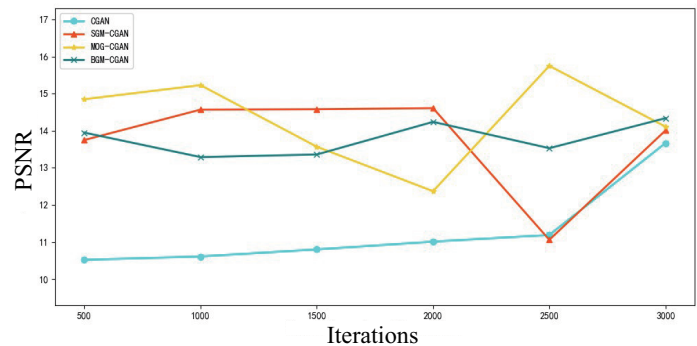

图 5 触觉 - 视觉跨模态 PSNR 值随迭代次数改变 结果

Figure 5 The tactile-visual cross-modal PSNR value changes with the number of iterations

算法迭代次数对跨模态生成图像质量的作用, 本次实验将各算法分别迭代 500 次、1000 次、1500 次、 2000 次、 2500 次和 3000 次, 比较其 PSNR 值, 如图 4 和 5 分别为视觉 - 触觉和触觉 - 视觉跨模态情 况下不同迭代次数 PSNR 值变化情况.

从图 4 和 5 的评估结果得知, 在迭代 3000 次之后, BGM-CGAN 算法相比其他算法取得的峰值 信噪比 PSNR 值最大, 说明该算法进行深度迭代相比于浅层次迭代更能将算法的优势体现出来, 并且 从 PSNR 值可以看出, 在双重跨模态生成图像过程中, BGM-CGAN 算法跨模态生成图像的效果更加 稳定, 而其他生成算法随着迭代次数的增加, 中间更易出现模型崩塌、损坏等情况 (从折线图中其他算 法在进行一定迭代次数后出现的最低峰值可以看出). 但是发现, 在进行浅层次迭代时, 对于视觉 - 触 觉跨模态生成图像, CGAN 算法也能取得较好的信噪比值, 这可能的原因是触觉模态图像本身并不复 杂, 容易用随机均匀噪声刻画; 而对于触觉 - 视觉跨模态生成图像, MOG-CGAN 算法明显优于其他两 种单一噪声网络, 这可能的原因是视觉模态图像与触觉模态图像像素值异构性很大, 用高斯混合噪声 更容易刻画视觉图像的复杂性特征.

\subsection{5 算法执行时间}

上述实验主要是针对各个算法生成的跨模态图像的质量、多样性、亮度、对比度和结构相似性等 方面的测试对比, 但算法执行时间也是考量算法的一个很重要的测定标准, 故接下来本文分别测试各 个生成式算法跨模态生成图像所需要的执行时间, 为了节省运行内存, 本文只对每个算法执行 100 次 迭代. 如表 4 所示为每个算法迭代所需要执行的时间, 总体来看, BGM-CGAN 算法时间复杂度与其他 算法差不多, 原因是产生多个高斯噪声组件并不需要太多时间, 故提出的算法满足运行要求.

\section{4 定性评估}

下面从定性分析角度进一步评估跨模态生成图像的质量, 观察是否符合真实图像特征. 本次实验 从 Gelsight-external grasp datasets 数据集中随机选取一个物体进行跨模态生成图像呈现, 每个物体选 定为抓取稳定操作 (抓取时物体没有发生相对滑动, 便于算法生成), 并只选择抓取物体操作最后 5 个 时步进行图像生成, 如图 6 所示为真实图像和各算法生成图像效果. BGM-CGAN 算法和 MOG-CGAN 算法对于触觉 - 视觉跨模态生成图像能力比较强, 这可能是由于视觉图像的像素复杂性需要混合噪 声组才能更容易刻画其相应特征; 在视觉 - 触觉跨模态生成图像能力评估中, BGM-CGAN 算法和 SGM-CGAN 算法生成效果比较好, 这可能是由于触觉图像的像素简易性, 所以 SGM-CGAN 算法也 
表 4 各算法运行时间对比 (min)

Table 4 Comparison of the running time (min) of various algorithms

\begin{tabular}{ccc}
\hline Algorithm & Visual-tactile & Tactile-visual \\
\hline CGAN & 20.2 & 20.2 \\
SGM-CGAN & 21.2 & 21.3 \\
MOG-CGAN & 21.7 & 21.7 \\
BGM-CGAN & 22.5 & 22.4 \\
\hline
\end{tabular}

表 5 被测试物体数据增强前后滑动检测结果对比

Table 5 Comparison of sliding detection results before and after data enhancing of test objects

\begin{tabular}{ccc}
\hline Object & Raw data $(\%)$ & Enhanced data $(\%)$ \\
\hline Object1 & 69.2 & 71.4 \\
Object2 & 54.5 & 58.3 \\
Object3 & 70.0 & 72.7 \\
Object4 & 66.7 & 68.4 \\
Object5 & 58.3 & 61.5 \\
Object6 & 64.2 & 66.7 \\
Object7 & 70.0 & 72.7 \\
Object8 & 63.6 & 66.7 \\
Object9 & 75.0 & 77.8 \\
Object10 & 52.9 & 55.6 \\
\hline Mean value & 64.4 & 67.2 \\
\hline
\end{tabular}

能较好生成其特征.

\section{5 模型评估}

前面通过各种评估方法验证了本文提出的 BGM-CGAN 算法模型在跨模态生成能力方面可以生 成与真实图像相差性较小的样本图像, 这为探索异构模态潜在表征特性和多模态融合数据增强研究提 供了相应的方法. 为了进一步探索其生成表现能力, 利用 BGM-CGAN 算法跨模态生成能力对滑动检 测数据集进行数据增强. 从 Gelsight-external grasp datasets 数据集中随机选择 40 个物体, 其中 30 个 物体被选定为训练对象, 另外 10 个物体为测试对象, 如图 7 所示, 蓝色圈内为选中的训练对象, 褐色 圈内为选中的测试对象. 本文将利用 BGM-CGAN 算法对每个测试对象的相应抓取稳定操作进行最 后 5 个时步的数据增强 (只对抓取稳定操作没有发生相对滑动的部分进行数据增强), 并利用多模态 ConvLSTM 算法进行滑动检测.

本文在每个模态中利用两层 ConvLSTM 网络进行滑动检测时空特征提取, 并最终在全连接层进 行特征融合, 如图 8 所示. 利用多模态 ConvLSTM 网络进行相应测试集的滑动检测结果预测, 结果如 表 5 所示.

从表 5 的结果得出, BGM-CGAN 算法可以有效地提升多模态融合表现, 通过该算法生成跨模态 数据对原始数据进行数据增强, 使原始数据滑动检测平均结果从 64.4 上升到 67.2 , 再次证明了本文提 出的 BGM-CGAN 跨模态生成式网络可以生成高还原度的跨模态图像. 

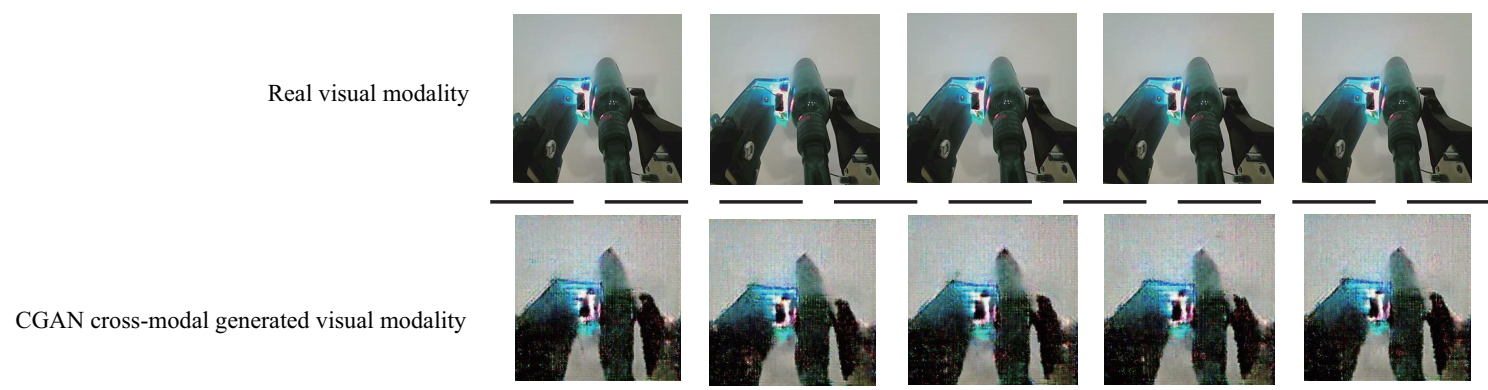

SGM-CGAN cross-modal generated visual modality
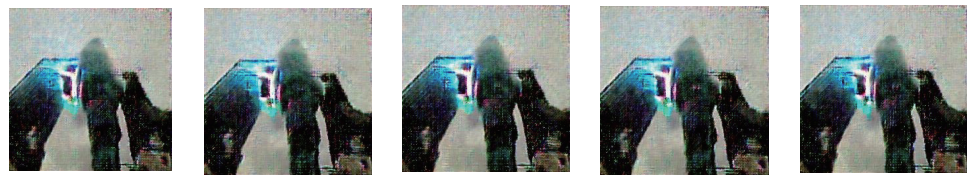

MOG-CGAN cross-modal generated visual modality
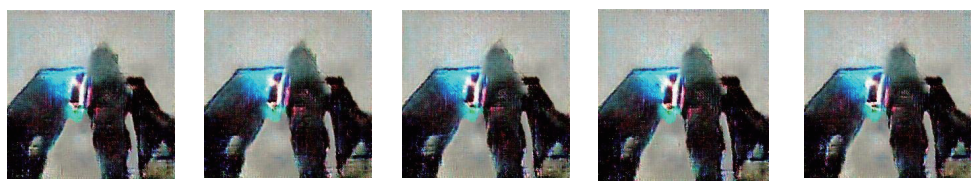

BGM-CGAN cross-modal generated visual modality
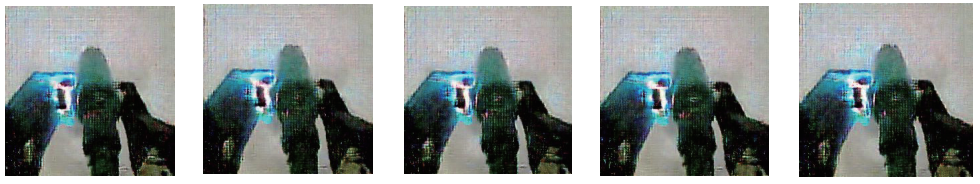

Real tactile modality
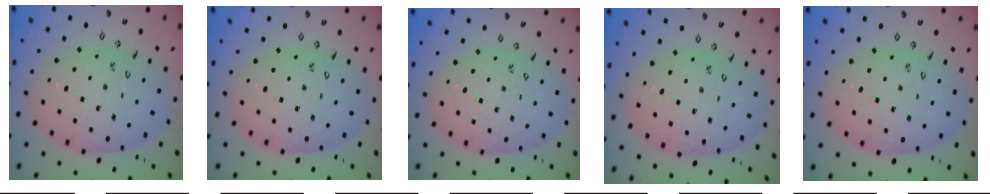

CGAN cross-modal generated tactile modality
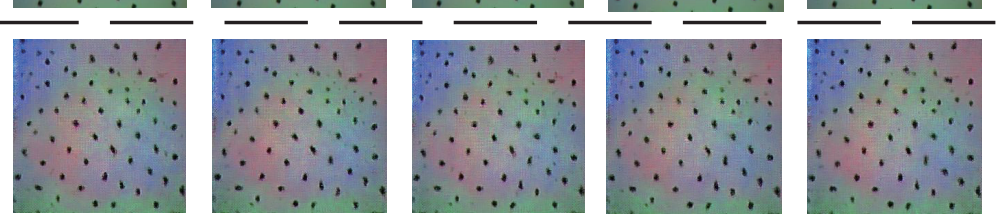

SGM-CGAN cross-modal generated tactile modality
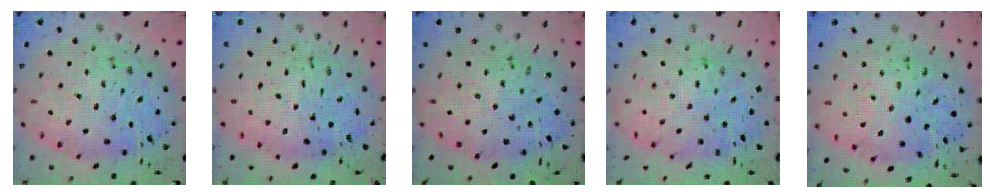

MOG-CGAN cross-modal generated tactile modality
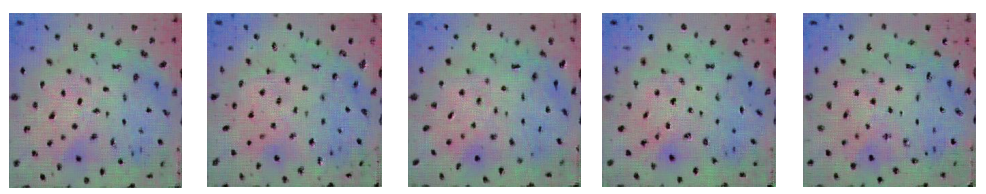

BGM-CGAN cross-modal generated tactile modality
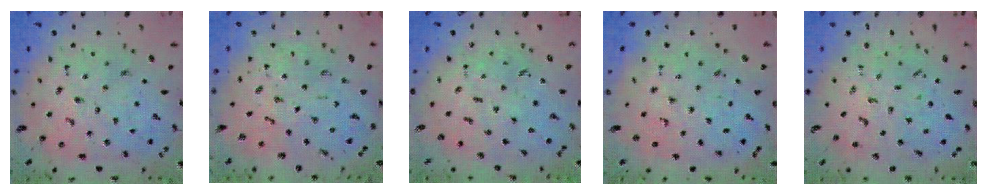

图 6 某个物体的跨模态生成图像与真实图像对比情况

Figure 6 Comparison of the cross-modal generated images with the real images of an object 


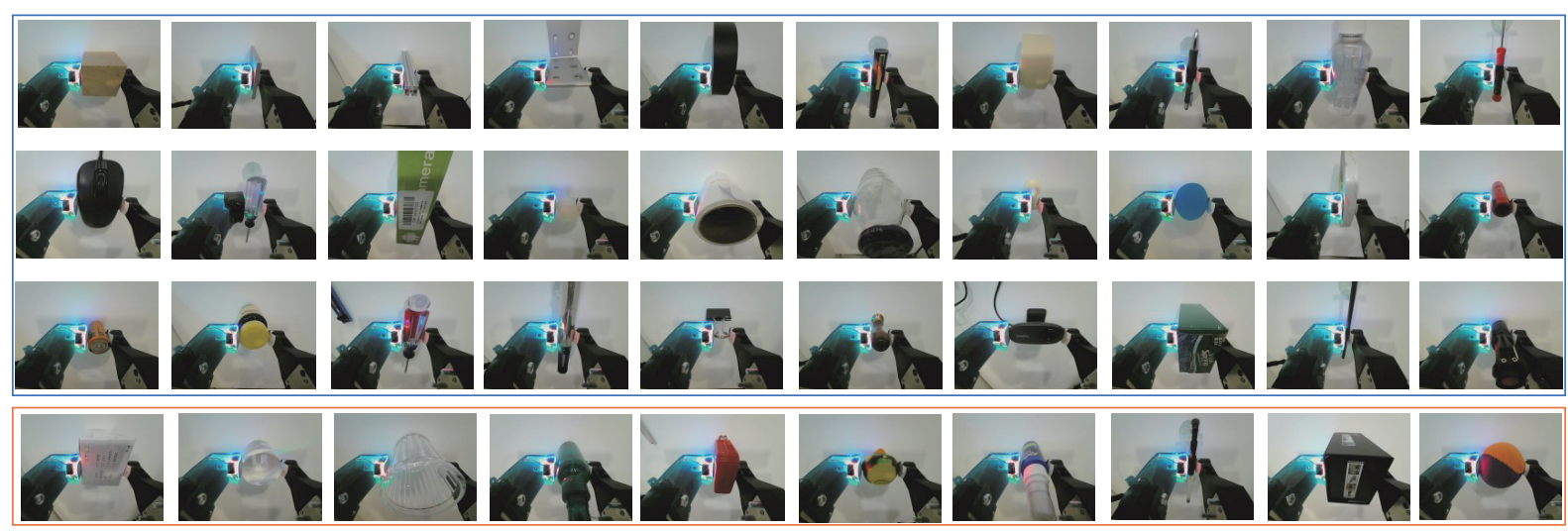

图 7 被选中的 40 个物体

Figure 7 Selected 40 objects

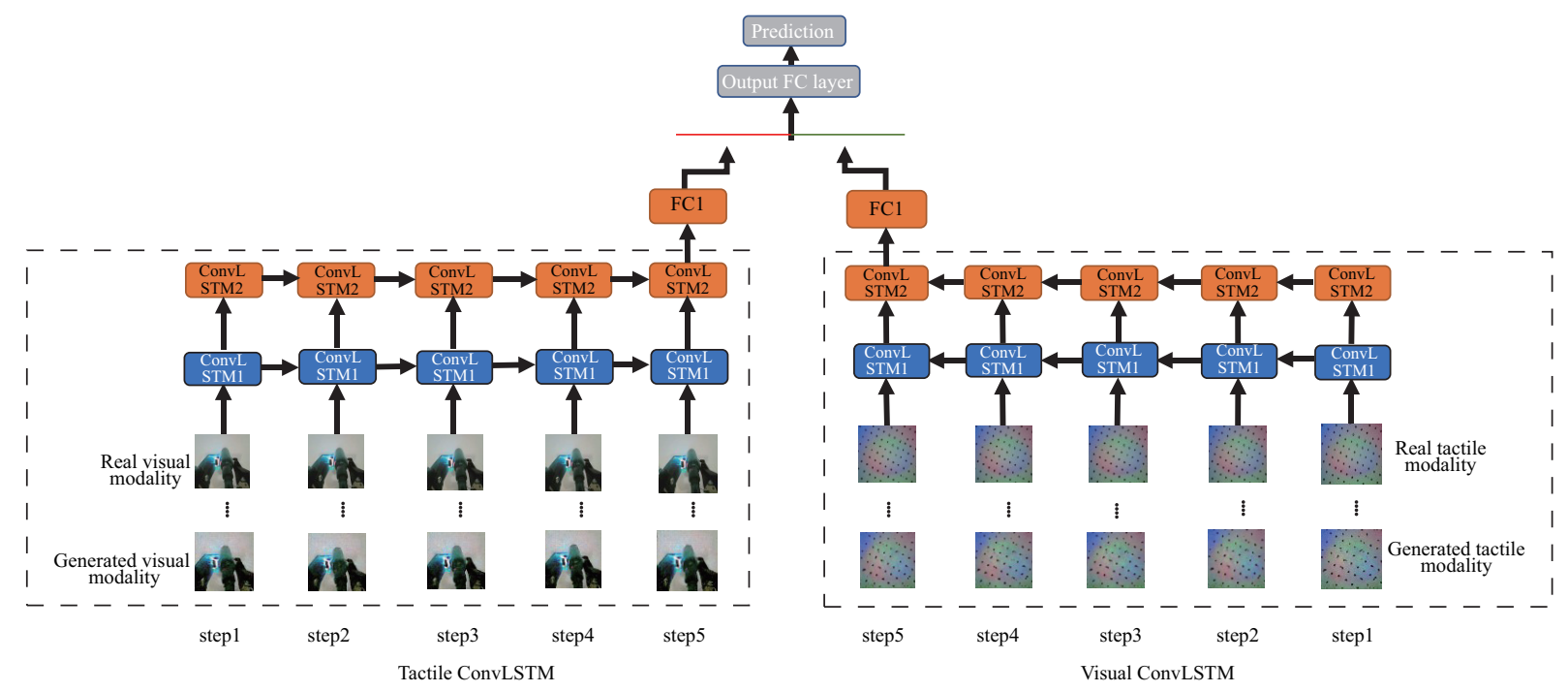

图 8 视觉 - 触觉融合 ConvLSTM 网络

Figure 8 Visual-tactile fusion ConvLSTM network

\section{6 结论}

本文针对传统的跨模态生成网络 CGAN 生成图像质量差, 容易出现图像崩塌、失真等问题, 提出 了一种基于变分贝叶斯高斯混合噪声的跨模态生成网络模型 BGM-CGAN, 该算法可以通过噪声的复 杂性刻画异构图像生成过程的像素特征, 生成图像具有高还原度、清晰度等特点, 并经多种评价体系 验证了 BGM-CGAN 跨模态生成能力. 另外, BGM-CGAN 算法还可延伸应用于跨模态材料检索、跨 模态纹理识别等领域, 具有广泛应用前景. 未来还会进一步探索触觉时间序列数据与视觉图像数据间 的跨模态高还原度生成算法以及具有时序变化特征的多种模态间的跨模态生成算法. 


\section{参考文献}

1 Liu H, Guo D, Sun F. Object recognition using tactile measurements: kernel sparse coding methods. IEEE Trans Instrum Meas, 2016, 65: 656-665

2 Liu H, Yu Y, Sun F, et al. Visual-tactile fusion for object recognition. IEEE Trans Automat Sci Eng, 2017, 14: 996-1008

3 Liu H, Sun F, Fang B, et al. Multimodal measurements fusion for surface material categorization. IEEE Trans Instrum Meas, 2018, 67: 246-256

4 Yuan W, Zhu C, Owens A, et al. Shape-independent hardness estimation using deep learning and a gelsight tactile sensor. In: Proceedings of IEEE International Conference on Robotics and Automation (ICRA), 2017. 951-958

$5 \mathrm{Su}$ J, Zhang Y Z, Fang L J, et al. Estimation of the grasping pose of unknown objects based on multiple geometric constraints. ROBOT, 2020, 42: 129-138 [苏杰, 张云洲, 房立金, 等. 基于多重几何约束的未知物体抓取位姿估计. 机器人, 2020, 42: 129-138]

6 Zhong X G, Xu M, Zhong X Y, et al. Multimodal features deep learning for robotic potential grasp recognition. Acta Autom Sin, 2016, 42: 1022-1029 [仲训杲, 徐敏, 仲训昱, 等. 基于多模特征深度学习的机器人抓取判别方法. 自动 化学报, 2016, 42: 1022-1029]

7 Garcia-Garcia A, Zapata-Impata B S, Orts-Escolano S, et al. TactileGCN: a graph convolutional network for predicting grasp stability with tactile sensors. In: Proceedings of International Joint Conference on Neural Networks (IJCNN), 2019. $1-8$

8 Zapata-Impata B S, Gil P, Torres F. Non-matrix tactile sensors: how can be exploited their local connectivity for predicting grasp stability? 2018. ArXiv: 1809.05551

9 Li J, Dong S, Adelson E. Slip detection with combined tactile and visual information. In: Proceedings of IEEE International Conference on Robotics and Automation (ICRA), 2018. 7772-7777

10 Calandra R, Owens A, Jayaraman D, et al. More than a feeling: learning to grasp and regrasp using vision and touch. IEEE Robot Autom Lett, 2018, 3: 3300-3307

11 Yuan W, Srinivasan M A, Adelson E H. Estimating object hardness with a gelsight touch sensor. In: Proceedings of IEEE/RSJ International Conference on Intelligent Robots and Systems (IROS), 2016. 208-215

12 Yuan W, Zhu C, Owens A, et al. Shape-independent hardness estimation using deep learning and a gelsight tactile sensor. In: Proceedings of IEEE International Conference on Robotics and Automation (ICRA), 2017. 951-958

13 Yuan W, Li R, Srinivasan M A, et al. Measurement of shear and slip with a GelSight tactile sensor. In: Proceedings of IEEE International Conference on Robotics and Automation (ICRA), 2015. 304-311

14 Jia X, Li R, Srinivasan M A, et al. Lump detection with a gelsight sensor. In: Proceedings of World Haptics Conference (WHC), 2013. 175-179

15 Li R, Adelson E H. Sensing and recognizing surface textures using a gelsight sensor. In: Proceedings of the IEEE Conference on Computer Vision and Pattern Recognition, 2013. 1241-1247

16 Gurumurthy S, Sarvadevabhatla R K, Babu R V. DeLiGAN: generative adversarial networks for diverse and limited data. In: Proceedings of the 30th IEEE Conference on Computer Vision and Pattern Recognition (CVPR), 2017. 4941-4949

17 Kingma D P, Welling M. Auto-encoding variational bayes. 2013. ArXiv: 1312.6114

18 Erickson Z, Chernova S, Kemp C C. Semi-supervised haptic material recognition for robots using generative adversarial networks. 2017. ArXiv: 1707.02796

19 Wei X, Li J, Sun X, et al. Sparse representation of robot image based on dictionary learning algorithm. Acta Autom Sin, 2020, 1-15. https://doi.org/10.16383/j.aas.c190743 [卫星, 李佳, 孙晓, 等. 基于混合生成对抗网络的多视角图 像生成算法. 自动化学报, 2020, 1-15. https://doi.org/10.16383/j.aas.c190743]

20 Li M Y, Zhou F Y, Tian T, et al. Design of the indoor WiFi cloud positioning system based on GAN and its application to service robots. ROBOT, 2018, 40: 693-703 [栗梦媛, 周风余, 田天, 等. 基于 GAN 的服务机器人室内 WiFi 云定 位系统设计与实现. 机器人, 2018, 40: 693-703]

21 Lee J T, Bollegala D, Luo S. "Touching to See" and "Seeing to Feel": robotic cross-modal sensory data generation for visual-tactile perception. In: Proceedings of International Conference on Robotics and Automation (ICRA), 2019. $4276-4282$

22 Falco P, Lu S, Cirillo A, et al. Cross-modal visuo-tactile object recognition using robotic active exploration. In: Proceedings of IEEE International Conference on Robotics and Automation (ICRA), 2017. 5273-5280

23 Zheng W, Liu H, Wang B, et al. Cross-modal surface material retrieval using discriminant adversarial learning. IEEE Trans Ind Inf, 2019, 15: 4978-4987 
24 Li Y, Zhu J Y, Tedrake R, et al. Connecting touch and vision via cross-modal prediction. In: Proceedings of the IEEE Conference on Computer Vision and Pattern Recognition (CVPR), 2019. 10609-10618

25 Li X, Liu H, Zhou J, et al. Learning cross-modal visual-tactile representation using ensembled generative adversarial networks. Cognitive Comput Syst, 2019, 99: 40-44

26 Luo S, Yuan W, Adelson E, et al. Vitac: feature sharing between vision and tactile sensing for cloth texture recognition. In: Proceedings of IEEE International Conference on Robotics and Automation (ICRA), 2018. 2722-2727

27 Liu H P, Sun F C, Fang B W, et al. Cross-modal zero-shot-learning for tactile object recognition. IEEE Trans Syst Man Cybern Syst, 2020, 50: 2466-2474

28 Tang X L, Du Y M, Liu Y W, et al. Image recognition with conditional deep convolutional generative adversarial networks. Acta Autom Sin, 2018, 44: 855-864 [唐贤伦, 杜一铭, 刘雨微, 等. 基于条件深度卷积生成对抗网络的图 像识别方法. 自动化学报, 2018, 44: 855-864]

29 Dumoulin V, Belghazi I, Poole B, et al. Adversarially learned inference. 2016. ArXiv: 1606.00704

30 Chen X, Duan Y, Houthooft R, et al. InfoGAN: interpretable representation learning by information maximizing generative adversarial nets. In: Proceedings of the 30th International Conference on Neural Information Processing Systems, 2016. 2172-2180

31 Yan S X, Li Y P, Feng X S. An AUV adaptive sampling method based on Gaussian process regression. ROBOT, 2019, 41: 232-241 [阎述学, 李一平, 封锡盛. 一种基于高斯过程回归的 AUV 自适应采样方法. 机器人, 2019, 41: 232-241]

\title{
Robotic cross-modal generative adversarial network based on vari- ational Bayesian Gaussian mixture noise model
}

\author{
Pengwen XIONG ${ }^{1,2,3^{*}}$, Xiaobao TONG ${ }^{1}$, Aiguo $\mathrm{SONG}^{2,3^{*}}$ \& Peter X. LIU ${ }^{4}$ \\ 1. School of Information Engineering, Nanchang University, Nanchang 330031, China; \\ 2. School of Instrument Science and Engineering, Southeast University, Nanjing 210096, China; \\ 3. State Key Laboratory of Bioelectronics, Southeast University, Nanjing 210096, China; \\ 4. Department of Systems and Computer Engineering, Carleton University, Ottawa K1S5B6, Canada \\ * Corresponding author. E-mail: steven.xpw@ncu.edu.cn, a.g.song@seu.edu.cn
}

\begin{abstract}
Multimodal fusion is essential for robots to fully perceive the external environment. Single modal information limits the ability of robots to recognize and grasp objects. Meanwhile, traditional cross-modal data generation methods produce poor images, resulting in the bad effects of multimodal fusion. To solve the problem of the poor image effects of multimodal generation and the lack of data for multimodal fusion, this study proposes a variational Bayesian Gaussian mixture-conditional generative adversarial network (BGM-CGAN) for generating diverse cross-modal noise data. With the variational Bayesian Gaussian mixture algorithm, a uniform distributed random noise group is generated into a single mixed variable. The generated mixed variable is then generated through a Gaussian mixture model to generate a series of Gaussian mixed noise groups. The generated mixed noise group is randomly selected into a single Gaussian noise and imported into a modal image and fused with the modal image to successfully generate a high-resolution heterogeneous modal image. The method restores the heterogeneous modal information and solves the problem of the insufficient information and poor quality of generated images under a single modal. In the end, we use a variety of evaluation indexes (IS, FID, SSIM, and PSNR) to compare the proposed BGM-CGAN and other algorithms for cross-modal image generation capabilities. Results show the effectiveness and feasibility of the proposed algorithm. In addition, the BGM-CGAN algorithm has wide application prospects and can be extended to cross-modal material retrieval, cross-modal texture recognition, and other fields.
\end{abstract}

Keywords cross-modal, variational Bayesian Gaussian mixture, conditional generative adversarial networks 


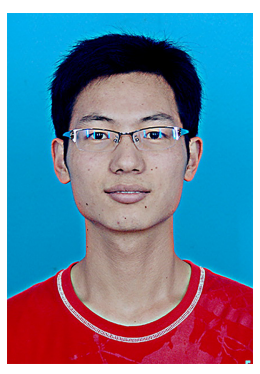

Pengwen XIONG received his B.S. degree from the North University of China in 2009 and his Ph.D. degree from the School of Instrument Science and Technology, Southeast University, China, in 2015. He visited the Laboratory for Computational Sensing and Robotics in Johns Hopkins University from 2013 to 2014. He is currently an associate professor at the School of Information Engineering of Nanchang University. He is also a postdoctoral researcher at the School of Instrument Science and Engineering, Southeast University. His research interests include humanrobot interaction and robotic sensing and control.

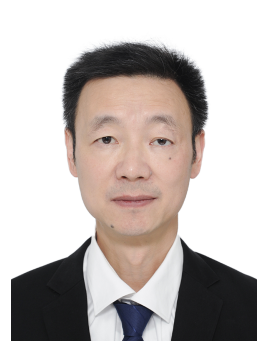

Aiguo SONG received his B.S. degree in automatic control and his M.S. degree in measurement and control from the Nanjing University of Aeronautics and Astronautics in 1990 and 1993, respectively. He received his Ph.D. degree in measurement and control from Southeast University in $1996 . \mathrm{He}$ is currently a professor at the School of Instrument Science and Engineering, Southeast University. His current interests include teleoperation, haptic display, Internet telerobotics, and distributed measurement systems.

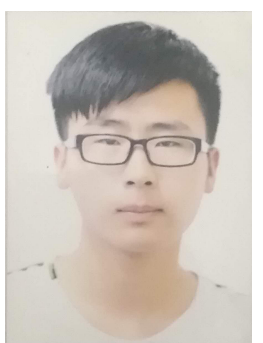

Xiaobao TONG received his B.E. degree in electrical engineering and automation from Chaohu University, China, in 2019. He is currently pursuing his master's degree in control science and engineering from Nanchang University, China.

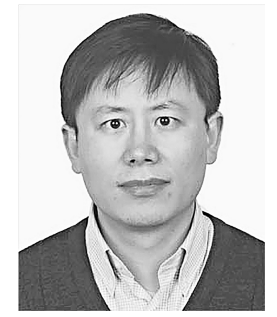

Peter X. LIU received his B.S. and M.S. degrees from Northern Jiaotong University, Beijing, China, in 1992 and 1995, respectively, and his Ph.D. degree from University of Alberta, Edmonton, AB, Canada, in 2002. He has been with the Department of Systems and Computer Engineering, Carleton University, Canada, since July 2002, and he is currently a research professor in Canada. He has been an IEEE Fellow since 2019. His research interests include interactive networked systems and teleoperations, robotics, intelligent systems, haptics, micromanipulation, context-aware intelligent networks, and their applications to biomedical engineering. 